\title{
Recurrence Coefficients of a New Generalization of the Meixner Polynomials ${ }^{\star}$
}

\author{
Galina FILIPUK ${ }^{\dagger}$ and Walter VAN ASSCHE ${ }^{\ddagger}$ \\ $\dagger$ Faculty of Mathematics, Informatics and Mechanics, University of Warsaw, \\ Banacha 2, Warsaw, 02-097, Poland \\ E-mail: filipuk@mimuw.edu.pl \\ URL: http://www.mimuw.edu.pl/ filipuk/ \\ $¥$ Department of Mathematics, Katholieke Universiteit Leuven, \\ Celestijnenlaan 200B box 2400, BE-3001 Leuven, Belgium \\ E-mail: walter@wis.kuleuven.be \\ URL: http://wis.kuleuven.be/analyse/walter/
}

Received April 18, 2011, in final form July 07, 2011; Published online July 13, 2011 doi:10.3842/SIGMA.2011.068

\begin{abstract}
We investigate new generalizations of the Meixner polynomials on the lattice $\mathbb{N}$, on the shifted lattice $\mathbb{N}+1-\beta$ and on the bi-lattice $\mathbb{N} \cup(\mathbb{N}+1-\beta)$. We show that the coefficients of the three-term recurrence relation for the orthogonal polynomials are related to the solutions of the fifth Painleve equation $\mathrm{P}_{\mathrm{V}}$. Initial conditions for different lattices can be transformed to the classical solutions of $\mathrm{P}_{\mathrm{V}}$ with special values of the parameters. We also study one property of the Bäcklund transformation of $\mathrm{P}_{\mathrm{V}}$.
\end{abstract}

Key words: Painlevé equations; Bäcklund transformations; classical solutions; orthogonal polynomials; recurrence coefficients

2010 Mathematics Subject Classification: 34M55; 33E17; 33C47; 42C05; 64Q30

\section{Introduction}

The recurrence coefficients of orthogonal polynomials for semi-classical weights are often related to the Painlevé type equations (e.g., [10, 4, 5] and see also the overview in [2]). In this paper we study a new generalization of the Meixner weight. The recurrence coefficients of the corresponding orthogonal polynomials can be viewed as functions of one of the parameters. We show that the recurrence coefficients are related to solutions of the fifth Painlevé equation. Another generalization of the Meixner weight is presented in [2].

The paper is organized as follows. In the introduction we shall first review orthogonal polynomials for the generalized Meixner weight on different lattices and their main properties following [12]. Next we shall briefly recall the fifth Painlevé equation and its Bäcklund transformation. Further, by using the Toda system, we show that the recurrence coefficients can be expressed in terms of solutions of the fifth Painlevé equation. Finally we study initial conditions of the recurrence coefficients for different lattices and describe one property of the Bäcklund transformation of $\mathrm{P}_{\mathrm{V}}$.

\footnotetext{
${ }^{\star}$ This paper is a contribution to the Special Issue "Relationship of Orthogonal Polynomials and Special Functions with Quantum Groups and Integrable Systems". The full collection is available at http://www.emis.de/journals/SIGMA/OPSF.html
} 


\subsection{Orthogonal polynomials for the generalized Meixner weight}

One of the most important properties of orthogonal polynomials is the three-term recurrence relation. Let us consider a sequence $\left(p_{n}\right)_{n \in \mathbb{N}}$ of orthonormal polynomials for the weight $w$ on the lattice $\mathbb{N}=\{0,1,2,3, \ldots\}$

$$
\sum_{k=0}^{\infty} p_{n}(k) p_{k}(k) w(k)=\delta_{n, k}
$$

where $\delta_{n, k}$ is the Kronecker delta. This relation takes the following form:

$$
x p_{n}(x)=a_{n+1} p_{n+1}(x)+b_{n} p_{n}(x)+a_{n} p_{n-1}(x) .
$$

For the monic polynomials $P_{n}$ related to orthonormal polynomials $p_{n}(x)=\gamma_{n} x^{n}+\cdots$ with

$$
\frac{1}{\gamma_{n}^{2}}=\sum_{k=0}^{\infty} P_{n}^{2}(k) w(k)
$$

the recurrence relation is given by

$$
x P_{n}(x)=P_{n+1}(x)+b_{n} P_{n}(x)+a_{n}^{2} P_{n-1}(x) .
$$

The classical Meixner polynomials ([3, Chapter VI], [12]) are orthogonal on the lattice $\mathbb{N}$ with respect to the negative binomial (or Pascal) distribution:

$$
\sum_{k=0}^{\infty} M_{n}(k ; \beta, c) M_{m}(k ; \beta, c) \frac{(\beta)_{k} c^{k}}{k !}=\frac{c^{-n} n !}{(\beta)_{n}(1-c)^{\beta}} \delta_{n, m}, \quad \beta>0, \quad 0<c<1 .
$$

Here the Pochhammer symbol is defined by

$$
(x)_{n}=\frac{\Gamma(x+n)}{\Gamma(x)}=x(x+1) \cdots(x+n-1) .
$$

The weight $w_{k}=w(k)=(\beta)_{k} c^{k} / k$ ! satisfies the Pearson equation

$$
\nabla[(\beta+x) w(x)]=\left(\beta+x-\frac{x}{c}\right) w(x),
$$

where $\nabla$ is the backward difference operator

$$
\nabla f(x)=f(x)-f(x-1)
$$

Here the function $w(x)=\Gamma(\beta+x) c^{x} /(\Gamma(\beta) \Gamma(x+1))$ gives the weights $w_{k}=w(k)$. The Pearson equation for the Meixner polynomials is, hence, of the form

$$
\nabla[\sigma(x) w(x)]=\tau(x) w(x)
$$

with $\sigma(x)=\beta+x$ and $\tau$ is a polynomial of degree 1 .

Recall that the classical orthogonal polynomials are characterized by the Pearson equation (2) with $\sigma$ a polynomial of degree at most 2 and $\tau$ a polynomial of degree 1 . Note that in (2) the operator $\nabla$ is used for orthogonal polynomials on the lattice and it is replaced by differentiation in case of orthogonal polynomials on an interval of the real line. The Pearson equation plays an important role for classical orthogonal polynomials since it allows to find many useful properties 
of these polynomials. It is known that the recurrence coefficients of the Meixner polynomials are given explicitly by

$$
a_{n}^{2}=\frac{n(n+\beta-1) c}{(1-c)^{2}}, \quad b_{n}=\frac{n+(n+\beta) c}{1-c}, \quad n \in \mathbb{N} .
$$

The Meixner weight can be generalized [12]. One can use the weight function

$$
w(x)=\frac{\Gamma(\beta) \Gamma(\gamma+x) c^{x}}{\Gamma(\gamma) \Gamma(\beta+x) \Gamma(x+1)}
$$

which gives the weight

$$
w_{k}=w(k)=\frac{(\gamma)_{k} c^{k}}{(\beta)_{k} k !}, \quad c, \beta, \gamma>0,
$$

on the lattice $\mathbb{N}$. The orthonormal polynomials $\left(p_{n}\right)_{n \in \mathbb{N}}$ for weight (3) satisfy

$$
\sum_{k=0}^{\infty} p_{n}(k) p_{m}(k) w_{k}=0, \quad n \neq m .
$$

The special case $\beta=1$ was studied in [2]. The case $\beta=\gamma$ gives the well-known Charlier weight. The case $\gamma=1$ corresponds to the classical Charlier weight on the shifted lattice $\mathbb{N}+1-\beta$.

Theorem 1 ([12, Theorem 3.1]). The recurrence coefficients in the three-term recurrence relation (1) for the orthonormal polynomials defined by (4), with respect to weight (3) on the lattice $\mathbb{N}$, satisfy

$$
a_{n}^{2}=n c-(\gamma-1) u_{n}, \quad b_{n}=n+\gamma-\beta+c-(\gamma-1) v_{n} / c,
$$

where

$$
\begin{aligned}
& \left(u_{n}+v_{n}\right)\left(u_{n+1}+v_{n}\right)=\frac{\gamma-1}{c^{2}} v_{n}\left(v_{n}-c\right)\left(v_{n}-c \frac{\gamma-\beta}{\gamma-1}\right), \\
& \left(u_{n}+v_{n}\right)\left(u_{n}+v_{n-1}\right)=\frac{u_{n}}{u_{n}-c n /(\gamma-1)}\left(u_{n}+c\right)\left(u_{n}+c \frac{\gamma-\beta}{\gamma-1}\right),
\end{aligned}
$$

with initial conditions

$$
a_{0}^{2}=0, \quad b_{0}=\frac{\gamma c}{\beta} \frac{M(\gamma+1, \beta+1, c)}{M(\gamma, \beta, c)},
$$

where $M(a, b, z)$ is the confluent hypergeometric function ${ }_{1} F_{1}(a ; b ; z)$.

The system (5), (6) can be identified as a limiting case of an asymmetric discrete Painlevé equation [12]. In this paper we show that it can be obtained from the Bäcklund transformation of the fifth Painlevé equation. Furthermore, one can use the weight (3) on the shifted lattice $\mathbb{N}+1-\beta$ and one can also combine both lattices to obtain the bi-lattice $\mathbb{N} \cup(\mathbb{N}+1-\beta)$. The orthogonality measure for the bi-lattice is a linear combination of the measures on $\mathbb{N}$ and $\mathbb{N}+1-\beta$.

The weight $w$ in (3) on the shifted lattice $\mathbb{N}+1-\beta=\{1-\beta, 2-\beta, 3-\beta, \ldots\}$ is, up to a constant factor, equal to the weight on the original lattice $\mathbb{N}$, with different parameters [12]. Denoting

$$
w_{\gamma, \beta, c}(x)=\frac{\Gamma(\beta)}{\Gamma(\gamma)} \frac{\Gamma(\gamma+x) c^{x}}{\Gamma(x+1) \Gamma(\beta+x)}
$$


one has

$$
w_{\gamma, \beta, c}(k+1-\beta)=c^{1-\beta} \frac{\Gamma(\beta) \Gamma(\gamma+1-\beta)}{\Gamma(2-\beta) \Gamma(\gamma)} w_{\gamma+1-\beta, 2-\beta, c}(k) .
$$

The corresponding orthonormal polynomials $\left(q_{n}\right)_{n \in \mathbb{N}}$ satisfy

$$
\sum_{k=0}^{\infty} q_{n}(k+1-\beta) q_{m}(k+1-\beta) w(k+1-\beta)=0, \quad n \neq m .
$$

Moreover, these polynomials are equal to the polynomials $p_{n}$ shifted in both the variable $x$ and the parameters $\beta$ and $\gamma$. For the positivity of the weights $(w(k+1-\beta))_{k \in \mathbb{N}}$ it is necessary to have $c>0, \beta<2, \gamma>\beta-1$. In [12, Theorem 3.2] it is shown that the recurrence coefficients in the three-term recurrence relation

$$
x q_{n}(x)=\hat{a}_{n+1} q_{n+1}(x)+\hat{b}_{n} q_{n}(x)+\hat{a}_{n} q_{n-1}(x)
$$

satisfy the same system (5), (6) (with hats) but with initial conditions

$$
\hat{a}_{0}^{2}=0, \quad \hat{b}_{0}=(1-\beta) \frac{M(\gamma-\beta+1,1-\beta, c)}{M(\gamma-\beta+1,2-\beta, c)} .
$$

Using the orthogonality measure $\mu=\mu_{1}+\tau \mu_{2}$, where $\tau>0, \mu_{1}$ is the discrete measure on $\mathbb{N}$ with weights $w_{k}=w(k)$ and $\mu_{2}$ is the discrete measure on $\mathbb{N}+1-\beta$ with weights $v_{k}=w(k+1-\beta)$, one can study orthonormal polynomials $\left(r_{n}\right)_{n \in \mathbb{N}}$, satisfying the three-term recurrence relation

$$
x r_{n}(x)=\tilde{a}_{n+1} r_{n+1}(x)+\tilde{b}_{n} r_{n}(x)+\tilde{a}_{n} r_{n-1}(x) .
$$

One needs to impose the conditions $c>0,0<\beta<2, \gamma>\max (0, \beta-1)$ for the positivity of the measures. The orthogonality relation is given by

$$
\sum_{k=0}^{\infty} r_{n}(k) r_{m}(k) w(k)+\tau \sum_{k=0}^{\infty} r_{n}(k+1-\beta) r_{m}(k+1-\beta) w(k+1-\beta)=0, \quad m \neq n
$$

According to [12, Theorem 3.3] the recurrence coefficients $\tilde{a}_{n}^{2}$ and $\tilde{b}_{n}$ satisfy system (5), (6) (with tilde) but with initial conditions

$$
\tilde{a}_{0}^{2}=0, \quad \tilde{b}_{0}=\frac{m_{1}+\tau \hat{m}_{1}}{m_{0}+\tau \hat{m}_{0}}
$$

where

$$
\begin{aligned}
& m_{0}=M(\gamma, \beta, c), \quad m_{1}=\frac{\gamma c}{\beta} M(\gamma+1, \beta+1, c), \\
& \hat{m}_{0}=\frac{\Gamma(\beta) \Gamma(\gamma-\beta+1)}{\Gamma(\gamma) \Gamma(2-\beta)} c^{1-\beta} M(\gamma-\beta+1,2-\beta, c), \\
& \hat{m}_{1}=\frac{\Gamma(\beta) \Gamma(\gamma-\beta+1)}{\Gamma(\gamma) \Gamma(1-\beta)} c^{1-\beta} M(\gamma-\beta+1,1-\beta, c) .
\end{aligned}
$$

Thus, it is shown in [12] that the orthogonal polynomials for the generalized Meixner weight on the lattice $\mathbb{N}$, on the shifted lattice $\mathbb{N}+1-\beta$ and on the bi-lattice $\mathbb{N} \cup(\mathbb{N}+1-\beta)$ have recurrence coefficients $a_{n}^{2}$ and $b_{n}$ which satisfy the same nonlinear system of discrete (recurrence) equations but the initial conditions are different in each case. 


\subsection{The fifth Painlevé equation and its Bäcklund transformation}

The Painlevé equations possess the so-called Painlevé property: the only movable singularities of the solutions are poles [9]. They are often referred to as nonlinear special functions and have numerous applications in mathematics and mathematical physics.

The fifth Painlevé equation $\mathrm{P}_{\mathrm{V}}$ is given by

$$
y^{\prime \prime}=\left(\frac{1}{2 y}+\frac{1}{y-1}\right)\left(y^{\prime}\right)^{2}-\frac{y^{\prime}}{t}+\frac{(y-1)^{2}}{t^{2}}\left(A y+\frac{B}{y}\right)+\frac{C y}{t}+\frac{D y(y+1)}{y-1},
$$

where $y=y(t)$ and $A, B, C, D$ are arbitrary complex parameters. By using a transformation $y(t) \rightarrow y\left(k_{1} t\right)$ we can take the value of the parameter $D$ equal to any non-zero number. There exists a Bäcklund transformation between solutions of the fifth Painlevé equation with $D \neq 0$.

Theorem 2 ([9, Theorem 39.1]). If $y=y(t)$ is the solution the fifth Painlevé equation (11) with parameters $A, B, C, D$, then the transformation

$$
T_{\varepsilon_{1}, \varepsilon_{2}, \varepsilon_{3}}: y \rightarrow y_{1}
$$

gives another solution $y_{1}=y_{1}(t)$ with new values of the parameters $A_{1}, B_{1}, C_{1}, D_{1}$, where

$$
\begin{aligned}
& y_{1}=1-\frac{2 d t y}{t y^{\prime}-a y^{2}+(a-b+d t) y+b}, \\
& A_{1}=-\frac{1}{16 D}(C+d(1-a-b))^{2}, \quad B_{1}=\frac{1}{16 D}(C-d(1-a-b))^{2}, \\
& C_{1}=d(b-a), \quad D_{1}=D, \\
& a=\varepsilon_{1} \sqrt{2 A}, \quad b=\varepsilon_{2} \sqrt{-2 B}, \quad d=\varepsilon_{3} \sqrt{-2 D}, \quad \varepsilon_{j}^{2}=1, \quad j \in\{1,2,3\} .
\end{aligned}
$$

See also [11] for a further description of the Bäcklund transformations and the isomorphism of the group of Bäcklund transformations and the affine Weyl group of $A_{3}^{(1)}$ type.

\section{Main results}

In this paper we show how to obtain a relation between the recurrence coefficients and the (classical) solutions of the fifth Painlevé equation. The calculations are similar to calculations in [2] but are more involved. The study of initial conditions of the recurrence coefficients for different lattices is also presented. We can summarize the known results and our recent findings concerning the (generalized) Meixner weights as follows.

The weight $(\beta)_{k} c^{k} /(k !), \beta>0,0<c<1$, is the classical Meixner weight and the recurrence coefficients are known explicitly. The weight $(\beta)_{k} c^{k} /(k !)^{2}, \beta>0, c>0$, is studied in [2] and the recurrence coefficients are related to classical solutions of $\mathrm{P}_{\mathrm{V}}$ with parameters $((\beta-$ $\left.1)^{2} / 2,-(\beta+n)^{2} / 2,2 n,-2\right)$. It is shown in this paper that the recurrence coefficients for the weight $(\gamma)_{k} c^{k} /\left(k !(\beta)_{k}\right), c, \beta, \gamma>0$, are related to the classical solutions of $\mathrm{P}_{\mathrm{V}}$ with parameters $\left.\left((\gamma-1)^{2} / 2,-(\gamma-\beta+n)^{2} / 2, k_{1}(\beta+n),-k_{1}^{2} / 2\right)\right), k_{1} \neq 0$.

\subsection{Relation to the fifth Painlevé equation and its Bäcklund transformation}

First we obtain a nonlinear discrete equation for $v_{n}(c)$. From equation (5) with $n$ and equation (6) with $n+1$ we eliminate $u_{n+1}$ by computing the resultant. Next, from the obtained equation and (6) with $n$ we eliminate $u_{n}$. As a result, we obtain a nonlinear discrete equation for $v_{n}(c)$ which we denote by

$$
F\left(v_{n-1}, v_{n}, v_{n+1}, c\right)=0 .
$$


The equation was obtained by using Mathematica ${ }^{1}$ but it is too long and too complicated too include here explicitly (all enquiries concerning computations can be sent to the first author). We shall show later on that equation (12) can in fact be obtained from the Bäcklund transformation of the fifth Painlevé equation.

Next we derive the differential equation for $v_{n}$. In [2] we have used the Toda system. Since the weight $w$ in (3) on the shifted lattice $\mathbb{N}+1-\beta=\{1-\beta, 2-\beta, 3-\beta, \ldots\}$ is, up to a constant factor, equal to the weight on the original lattice $\mathbb{N}$ with different parameters [12], it can be shown $[2,5]$ that the recurrence coefficients $a_{n}$ and $b_{n}$ as functions of the parameter $c$ satisfy the Toda system

$$
\begin{aligned}
& \left(a_{n}^{2}\right)^{\prime}:=\frac{d}{d c}\left(a_{n}^{2}\right)=\frac{a_{n}^{2}}{c}\left(b_{n}-b_{n-1}\right), \\
& b_{n}^{\prime}:=\frac{d}{d c} b_{n}=\frac{1}{c}\left(a_{n+1}^{2}-a_{n}^{2}\right) .
\end{aligned}
$$

The same system (13) holds for the initial lattice $\mathbb{N}[2]$.

Solving (5) for $u_{n+1}$ and (6) for $v_{n-1}$ and substituting into the Toda system (13) (where we have replaced $a_{n}^{2}$ and $b_{n}$ by their expressions in terms of $u_{n}$ and $v_{n}$ from Theorem 1), we get two equations

$$
u_{n}^{\prime}=R_{1}\left(u_{n}, v_{n}, c\right)
$$

and

$$
v_{n}^{\prime}=R_{2}\left(u_{n}, v_{n}, c\right),
$$

where the differentiation is with respect to $c$. The explicit expressions for $R_{1}$ and $R_{2}$ are again quite complicated but readily computed in Mathematica. By differentiating equation (14) and substituting the expression for $u_{n}^{\prime}$ we obtain an equation for $v_{n}^{\prime \prime}, v_{n}^{\prime}, v_{n}, u_{n}$. Finally, eliminating $u_{n}$ between this equation and (14) gives a nonlinear second order second degree equation for $v_{n}$ :

$$
G\left(v_{n}^{\prime \prime}, v_{n}^{\prime}, v_{n}, c\right)=0 .
$$

We have again used Mathematica to compute this long expression. Now the main difficulty is in identifying this equation. Since (5), (6) is similar to the discrete system in [2], we can try to reduce equation (15) to the fifth Painlevé equation. First, we scale the independent variable $c \rightarrow c / k_{1}$ and denote $c=k_{1} t, v(c)=V(t)$. The equation (15) becomes $G_{1}\left(V_{n}^{\prime \prime}, V_{n}^{\prime}, V_{n}, t\right)=0$, where the differentiation is with respect to $t$. By considering the Ansatz

$$
V_{n}(t)=\frac{p_{1}(t) y^{\prime}+p_{2}(t) y^{2}+p_{3}(t) y+p_{4}(t)}{y(y-1)},
$$

where $y=y(t)$ is the solution of the fifth Painlevé equation and $p_{j}(t)$ are unknown functions, we finally get the following theorem.

Theorem 3. The equation $G_{1}\left(V_{n}^{\prime \prime}, V_{n}^{\prime}, V_{n}, t\right)=0$ is reduced to the fifth Painlevé equation $\mathrm{P}_{\mathrm{V}}$ by the following transformations:

1.

$$
V(t)=\frac{k_{1} t\left(t y^{\prime}-(1+\beta-2 \gamma) y^{2}+\left(1+n-k_{1} t+\beta-2 \gamma\right) y-n\right)}{2(\gamma-1)(y-1) y},
$$

where $y=y(t)$ satisfies $\mathrm{P}_{\mathrm{V}}$ with

$$
A=\frac{(\beta-1)^{2}}{2}, \quad B=-\frac{n^{2}}{2}, \quad C=k_{1}(n-\beta+2 \gamma), \quad D=-\frac{k_{1}^{2}}{2} ;
$$

\footnotetext{
${ }^{1}$ http://www. wolfram.com
} 
2.

$$
V(t)=\frac{k_{1} t\left(t y^{\prime}-(\beta-\gamma) y^{2}+\left(n-1-k_{1} t+\beta\right) y+1-n-\gamma\right)}{2(\gamma-1)(y-1) y},
$$

where $y=y(t)$ satisfies $\mathrm{P}_{\mathrm{V}}$ with

$$
A=\frac{(\beta-\gamma)^{2}}{2}, \quad B=-\frac{(\gamma+n-1)^{2}}{2}, \quad C=k_{1}(2+n-\beta), \quad D=-\frac{k_{1}^{2}}{2}
$$

3.

$$
V(t)=\frac{k_{1} t\left(t y^{\prime}+(\gamma-1) y^{2}+\left(1+n-k_{1} t-\beta\right) y-n+\beta-\gamma\right)}{2(\gamma-1)(y-1) y},
$$

where $y=y(t)$ satisfies $\mathrm{P}_{\mathrm{V}}$ with

$$
A=\frac{(\gamma-1)^{2}}{2}, \quad B=-\frac{(\gamma-\beta+n)^{2}}{2}, \quad C=k_{1}(\beta+n), \quad D=-\frac{k_{1}^{2}}{2} .
$$

Remark 1. The parameters (17) are invariant under $\beta \rightarrow 2-\beta, \gamma \rightarrow \gamma+1-\beta$; compare with the parameters in the weight (8).

Remark 2. Cases 2 and 3 in Theorem 3 follow from case 1 by considering the Bäcklund transformation of Theorem 2. Indeed, the compositions of the transformations

$$
T_{1,1,-1} \circ T_{1,-1,1}=T_{1,1,1} \circ T_{1,-1,-1}
$$

give the transformation

$$
Y_{1}=y-\frac{2(\gamma-1)(y-1)^{2} y}{t y^{\prime}-(1+\beta-2 \gamma) y^{2}+\left(1+n-k_{1} t+\beta-2 \gamma\right) y-n},
$$

where $y=y(t)$ solves $\mathrm{P}_{\mathrm{V}}$ with parameters (17) and $Y_{1}$ solves $\mathrm{P}_{\mathrm{V}}$ with (19). Similarly, the compositions of the transformations

$$
T_{1,1,-1} \circ T_{-1,-1,1}=T_{1,1,1} \circ T_{-1,-1,-1}
$$

give

$$
Y_{2}=w+\frac{2(\beta-\gamma)(y-1)^{2} y}{t y^{\prime}-(1+\beta-2 \gamma) y^{2}+\left(1+n-k_{1} t+\beta-2 \gamma\right) y-n},
$$

where $y=y(t)$ solves $\mathrm{P}_{\mathrm{V}}$ with $(17)$ and $Y_{2}$ solves $\mathrm{P}_{\mathrm{V}}$ with (20).

Next we show that equation (12) can, in fact, be obtained from the Bäcklund transformation of $\mathrm{P}_{\mathrm{V}}$ in Theorem 2. Let us take, for instance, the parameters (17) and $k_{1}=1$ for simplicity (hence, $c=t$ ). Suppose $y=y_{n}(t)$ solves $\mathrm{P}_{\mathrm{V}}$ with $(17)$. Then by considering the transformations $T_{1,-1,-1} \circ T_{-1,-1,1} \circ T_{-1,-1,1}$ and $T_{1,-1,-1} \circ T_{1,1,-1} \circ T_{1,1,1}$ we get new solutions of $\mathrm{P}_{\mathrm{V}}$ with parameters (17) for $n+1$ and $n-1$ respectively. In particular,

$$
\begin{aligned}
y_{n+1}= & 1-\frac{2 t(n+\gamma) y}{(\beta-1)\left(t y^{\prime}+y(1+n+t-\beta+(\beta-1) y)-n\right)} \\
& +\frac{2 t(1+n-\beta+\gamma) y}{(\beta-1)\left(t y^{\prime}+y(n-1+t+\beta+(1-\beta) y)-n\right)}
\end{aligned}
$$

and

$$
\begin{aligned}
y_{n-1}= & 1+\frac{2 t(\gamma+n-1) y}{(\beta-1)\left(t y^{\prime}-y(1+n+t-\beta+(\beta-1) y)+n\right)} \\
& -\frac{2 t(n-\beta+\gamma) y}{(\beta-1)\left(t y^{\prime}-y(n-1+t+\beta+(1-\beta) y)+n\right)} .
\end{aligned}
$$

Expressing $v_{n \pm 1}$ in terms of $y_{n}$ by using (16), we can compute that (12) is identically zero. Hence, the discrete system (5), (6) can be obtained from the Bäcklund transformation of $\mathrm{P}_{\mathrm{V}}$. 


\section{$2.2 \quad$ Initial conditions}

In this section we study the initial conditions (7), (9), (10) for the generalized Meixner weight (3) on the lattice $\mathbb{N}$, on the shifted lattice and on the bi-lattice respectively.

Let $n=0$. Since $\gamma \neq 1$, we get that $u_{0}=0$ from $a_{0}^{2}=0$. We also put $k_{1}=1$ and $c=t$. From (14) we have that $v=v_{0}(t)$ satisfies the first order nonlinear equation

$$
t^{2} v^{\prime}=(\gamma-1) v^{2}+t(2-t+\beta-2 \gamma) v+(\gamma-\beta) t^{2}
$$

Since $b_{0}=\gamma-\beta+t-(\gamma-1) v_{0} / t$, we can find $v_{0}$ for $(7),(9)$ and (10). We can verify that all of them satisfy (21) using formulas for the confluent hypergeometric functions from [1]. Note that (10) depends on an arbitrary parameter $\tau$.

The fifth Painlevé equation (11) with parameters (19) with $k_{1}=1$ and $n=0$ has particular solutions which solve the following first order nonlinear equation:

$$
t y^{\prime}=(\beta-\gamma) y^{2}+(t-1-\beta+2 \gamma) y+1-\gamma
$$

Substituting expression (18) in (21) and assuming that $y$ satisfies (22), we indeed find that the equation is satisfied. We also find that $v(t)=t / y(t)$. Thus, the initial conditions (7), (9), (10) for the generalized Meixner weight (3) on the lattice $\mathbb{N}$, on the shifted lattice and on the bi-lattice respectively are related to solutions of the first order differential equation (22), which, in turn, satisfies $\mathrm{P}_{\mathrm{V}}$.

\section{A remark on the Bäcklund transformation}

In this section we study the Bäcklund transformation of the fifth Painlevé equation $\left(\mathrm{P}_{\mathrm{V}}\right)$ and find when a linear combination of two solutions is also a solution of $\mathrm{P}_{\mathrm{V}}$. In particular, we show that if $y_{1}$ and $y_{2}$ are solutions of $\mathrm{P}_{\mathrm{V}}$ obtained from a solution $y$ by certain Bäcklund transformations, then there is a constant $M \neq 0,1$ such that $M y_{1}+(1-M) y_{2}$ is also a solution of $\mathrm{P}_{\mathrm{V}}$.

Example 1. In [2] it is shown that if $y:=y_{n}(t)$ (related to the recurrence coefficients of the generalized Meixner polynomials) is the solution of (11) with

$$
A=\frac{(\beta-1)^{2}}{2}, \quad B=-\frac{(\beta+n)^{2}}{2}, \quad C=2 n, \quad D=-2,
$$

then one can show that $y_{n+1}=y_{n+1}(t)$ given by

$$
\begin{aligned}
y_{n+1}= & +\frac{4(n+1) t y}{(\beta-1)\left(t y^{\prime}+(2 t+2 \beta-1+n-(\beta-1) y) y-n-\beta\right)} \\
& -\frac{4 t(n+\beta) y}{(\beta-1)\left(t y^{\prime}+(1+n+2 t+(\beta-1) y) y-n-\beta\right)}
\end{aligned}
$$

is the solution of (11) with

$$
A=\frac{(\beta-1)^{2}}{2}, \quad B=-\frac{(\beta+n+1)^{2}}{2}, \quad C=2(n+1), \quad D=-2 .
$$

It can be observed that the transformation (23) can be written in the following form:

$$
y_{n+1}=M y_{1}+(1-M) y_{2}, \quad M=\frac{n+1}{1-\beta},
$$


where

$$
y_{1}=T_{1,-1,1} y=1+\frac{4 t y}{n+\beta-(n-1+2 \beta+2 t) y+(\beta-1) y^{2}-t y^{\prime}}
$$

is a solution of (11) with

$$
A_{1}=\frac{(n+1)^{2}}{2}, \quad B_{1}=-\frac{1}{2}, \quad C_{1}=-2(2 \beta+n-1), \quad D_{1}=-2
$$

and

$$
y_{2}=T_{-1,-1,1} y=1+\frac{4 t y}{n+\beta-(1+n+2 t) y-(\beta-1) y^{2}-t y^{\prime}}
$$

is a solution of (11) with

$$
A_{2}=\frac{(n+\beta)^{2}}{2}, \quad B_{2}=-\frac{\beta^{2}}{2}, \quad C_{2}=-2(n+1), \quad D_{2}=-2 .
$$

Similarly, if

$$
\begin{aligned}
& y_{1}=T_{1,1,-1} y=1+\frac{4 t y}{n+\beta-(1+n+2 t) y-(\beta-1) y^{2}+t y^{\prime}}, \\
& y_{2}=T_{-1,1,-1} y=1+\frac{4 t y}{n+\beta-(n-1+2 \beta+2 t) y+(\beta-1) y^{2}+t y^{\prime}}
\end{aligned}
$$

are solutions of (11) with

$$
A_{1}=\frac{(\beta+n-1)^{2}}{2}, \quad B_{1}=-\frac{(\beta-1)^{2}}{2}, \quad C_{1}=-2(n+1), \quad D_{1}=-2
$$

and

$$
A_{2}=\frac{n^{2}}{2}, \quad B_{2}=0, \quad C_{2}=-2(2 \beta+n-1), \quad D_{2}=-2,
$$

respectively, then

$$
y_{n-1}=M y_{1}+(1-M) y_{2}, \quad M=\frac{(\beta+n-1)}{\beta-1}
$$

is a solution of (11) with

$$
A=\frac{(\beta-1)^{2}}{2}, \quad B=-\frac{(\beta+n-1)^{2}}{2}, \quad C=2(n-1), \quad D=-2 .
$$

Such observations motivate us to study the question when the sum $M y_{1}+(1-M) y_{2}$ of two solutions of $\mathrm{P}_{\mathrm{V}}$ is also a solution of the same equation. Clearly, we impose the conditions that $M \neq 0$ and $M \neq 1$.

Theorem 4. Let $y=y(t)$ be a solution of $P_{\mathrm{V}}$ with parameters $A, B, C, D=-2$ and

$$
y_{1}=T_{\varepsilon_{1}, \varepsilon_{2}, \varepsilon_{3}} y, \quad y_{2}=T_{\delta_{1}, \delta_{2}, \delta_{3}} y,
$$

where $\varepsilon_{j}^{2}=\delta_{j}^{2}=1$ and $\varepsilon_{j} \neq \delta_{j}$ for some $j \in\{1,2,3\}$. Then

$$
v=M y_{1}+(1-M) y_{2}
$$

with $M \neq 0 ; 1$ is a solution of $\mathrm{P}_{\mathrm{V}}$ with parameters $A_{v}, B_{v}, C_{v}, D_{v}=-2$ in the following cases: 
1. $\delta_{1}=\varepsilon_{1}, \delta_{2}=-\varepsilon_{2}, \delta_{3}=\varepsilon_{3}$ and

$$
\begin{aligned}
& M=\frac{2 \varepsilon_{1} \sqrt{2 A}+2 \varepsilon_{2} \sqrt{-2 B}-\varepsilon_{3} C-2}{4 \varepsilon_{2} \sqrt{-2 B}}, \\
& A_{v}=-B, \quad B_{v}=\frac{2 \varepsilon_{1} \sqrt{2 A}-2 A-1}{2}, \quad C_{v}=-C-2 \varepsilon_{3} ;
\end{aligned}
$$

2. $\delta_{1}=-\varepsilon_{1}, \delta_{2}=\varepsilon_{2}, \delta_{3}=\varepsilon_{3}$ and

$$
\begin{aligned}
& M=\frac{2 \varepsilon_{1} \sqrt{2 A}+2 \varepsilon_{2} \sqrt{-2 B}-\varepsilon_{3} C-2}{4 \varepsilon_{1} \sqrt{2 A}}, \\
& A_{v}=A, \quad B_{v}=\frac{2 \varepsilon_{2} \sqrt{-2 B}+2 B-1}{2}, \quad C_{v}=C+2 \varepsilon_{3} .
\end{aligned}
$$

Proof. The proof of this result is computational. We first obtain that in case $\delta_{3}=-\varepsilon_{3}$ one gets only cases $M=0$ and $M=1$. In case $\delta_{3}=\varepsilon_{3}$ one needs to consider 3 cases separately (since $\delta_{1}=\varepsilon_{1}, \delta_{2}=\varepsilon_{2}$ gives a trivial result for the function $v$ ): $\delta_{1}=\varepsilon_{1}, \delta_{2}=-\varepsilon_{2} ; \delta_{1}=-\varepsilon_{1}$, $\delta_{2}=\varepsilon_{2} ; \delta_{1}=-\varepsilon_{1}, \delta_{2}=-\varepsilon_{2}$. However, in the last case we get $M=0$ or $M=1$.

The examples at the beginning of the section correspond to the second case of the theorem. Similarly, we can get expressions for $y_{n+1}$ and $y_{n-1}$ in the previous section by using this theorem.

Various properties of the repeated application of the Bäcklund transformations are studied in $[6,7,8]$. Repeated applications of the Bäcklund transformations to the seed solutions usually lead to very cumbersome formulas. However, as shown in this section, we can get linear dependence between three solutions. Moreover, our formulas suggest that the function $v$ has the same poles as $y_{1}$ and $y_{2}$ and, thus, they can be useful to study various properties of the solutions. Other Painlevé equations might have similar properties so one can try to study when for instance a linear combination of several solutions or a product or a cross-ratio of several solutions is also a solution (see also the representation of solutions in [11]). Although computationally difficult, this deserves further study.

\section{Acknowledgements}

GF is partially supported by Polish MNiSzW Grant N N201 397937 . WVA is supported by Belgian Interuniversity Attraction Pole P6/02, FWO grant G.0427.09 and K.U. Leuven Research Grant OT/08/033.

\section{References}

[1] Abramowitz M., Stegun I., Handbook of mathematical functions, Dover Publications, New York, 1965.

[2] Boelen L., Filipuk G., Van Assche W., Recurrence coefficients of generalized Meixner polynomials and Painlevé equations, J. Phys. A: Math. Theor. 44 (2011), 035202, 19 pages.

[3] Chihara T.S., An introduction to orthogonal polynomials, Mathematics and its Applications, Vol. 13, Gordon and Breach Science Publishers, New York - London - Paris, 1978.

[4] Filipuk G., Van Assche W., Zhang L., On the recurrence coefficients of semiclassical Laguerre polynomials, arXiv:1105.5229.

[5] Filipuk G., Van Assche W., Recurrence coefficients of the generalized Charlier polynomials and the fifth Painlevé equation, arXiv:1106.2959.

[6] Gromak V., Filipuk G., On functional relations between solutions of the fifth Painlevé equation, Differ. Equ. 37 (2001), 614-620.

[7] Gromak V., Filipuk G., The Bäcklund transformations of the fifth Painleve equation and their applications, Math. Model. Anal. 6 (2001), 221-230. 
[8] Gromak V., Filipuk G., Bäcklund transformations of the fifth Painlevé equation and their applications, in Proceedings of the Summer School "Complex Differential and Functional Equations" (Mekrijärvi, 2000), Univ. Joensuu Dept. Math. Rep. Ser., Vol. 5, Univ. Joensuu, Joensuu, 2003, 9-20.

[9] Gromak V.I., Laine I., Shimomura S., Painlevé differential equations in the complex plane, de Gruyter Studies in Mathematics, Vol. 28, Walter de Gruyter \& Co., Berlin, 2002.

[10] Magnus A.P., Painlevé type differential equations for the recurrence coefficients of semi-classical orthogonal polynomials, J. Comput. Appl. Math. 57 (1995), 215-237, math.CA/9307218.

[11] Noumi M., Painlevé equations through symmetry, Translations of Mathematical Monographs, Vol. 223, American Mathematical Society, Providence, RI, 2004.

[12] Smet C., Van Assche W., Orthogonal polynomials on a bi-lattice, Constr. Approx., to appear, arXiv:1101.1817. 